\title{
Can renal dimensions and the main renal artery diameter indicate the presence of an accessory renal artery? A 64-slice CT study
}

\author{
Selma Uysal Ramadan, Hasan Yiğit, Dilek Gökharman, Işıl Tunçbilek, N. Anıl Dolgun, Pınar Koşar, Uğur Koşar
}

\begin{abstract}
PURPOSE
To evaluate whether renal dimensions and the main renal artery (mRA) diameter can provide indirect evidence of the presence or absence of accessory renal arteries (aRA).

MATERIALS AND METHODS

The study group consisted of 167 patients ( 83 women, 84 men; mean age, $52.4 \pm 13.1$ years) who presented to our radiology department for abdominal CT examinations with various indications. CT examination was performed on a 64-slice CT scanner in the arterial phase. The kidney diameters were recorded. The number of renal arteries supplying each kidney was evaluated, and their diameters were measured. We attempted to determine a formula that could be used to predict the presence or absence of aRAs.

RESULTS

One or multiple aRAs were found in $76(22.8 \%)$ of the 334 kidneys. The mRA diameter was $5.51 \pm 0.96 \mathrm{~mm}$. The $\mathrm{mRA}$ diameter was smaller in kidneys with aRAs than in those without $(P<0.001)$. A cut-off value of $4.15 \mathrm{~mm}$ for the diameter of mRA to predict the presence of aRAs led to negative and positive predictive values of $80 \%$ and $90 \%$, respectively. A formula that can predict that an aRA is absent with $97 \%$ accuracy given the values of the mRA diameter and the kidney length was determined using logistic regression.

CONCLUSION

The mRA diameter $(4.15 \mathrm{~mm}$ ) alone and the results of the formula developed herein employing the mRA diameter and kidney length can respectively predict the presence or absence of an aRA with high accuracy.
\end{abstract}

Key words: $\bullet$ kidney $\bullet$ renal artery $\bullet$ computed tomography $\bullet$ hypertension, renovascular

From the Department of Radiology (S.U.R. $\square$ uysalselma@yahoo. com, H.Y., D.G., I.T., P.K., U.K.), Ankara Training and Research Hospital, Ankara, Turkey; and the Department of Biostatistics (N.A.D.), Hacettepe University, Faculty of Medicine, Ankara, Turkey.

Received 11 April 2010; accepted 26 May 2010.

Published online 9 August 2010

DOI 10.4261/1305-3825.DIR.3507-10.0
$\mathbf{T}$ he accessory renal artery (aRA) is the most common and clinically important variation of the renal artery. The aRA arises as a separate branch from the aorta or iliac artery, and it has been reported with a prevalence of approximately $25-30 \%$ in different study groups (1-4). Determination of the presence of an aRA has major importance in invasive renal procedures, such as laparoscopic or retroperitoneoscopic nephrectomy for living donor kidney transplantation, in the prevention of complications during the procedure or in the preoperative evaluation of patients with renal tumors in whom nephron-sparing surgery can be performed, and finally in the evaluation of renovascular hypertension related to isolated aRA stenosis (4-8).

Renal vessels can be examined by several radiological modalities such as color Doppler ultrasound (CDUS), conventional angiography, computed tomography (CT) and magnetic resonance (MR) angiography. CDUS is frequently preferred because it is an easily accessible, cheap and non-invasive method. However, CDUS is frequently inadequate for the detection of an aRA because of major disadvantages such as operator dependency and technical difficulties due to patient obesity and abdominal gas artifacts (9). Conventional angiography, although an invasive imaging technique, has been accepted as the "gold standard" for evaluating renal vascularity (9). On the other hand, its implementation in routine clinical practice is limited due to its invasive nature. CT and especially multi-slice computed tomography (MSCT) have a growing importance in the evaluation of renal vessels (10). MSCT has advantages, such as the capacity to show the morphology of the kidney and other surrounding structures while evaluating the kidney vasculature noninvasively in a short period. MSCT has a narrow collimation, high spatial and temporal resolutions and nearly isotropic acquisition, and thus, it provides multiplanar imaging. In addition, its level of accuracy is similar to conventional angiography in evaluating renal vascularity $(6,7,10)$. On the other hand, the disadvantages of MSCT are radiation exposure and the dependence on contrast medium. MR angiography is another non-invasive imaging technique that is used for the evaluation of renal vascularity $(11,12)$. Recent studies of gadolinium-enhanced MR angiography have demonstrated its higher rates of accuracy, which approach those of conventional angiography and CT angiography in the evaluation of living kidney donors for nephrectomy (11-13). MR imaging has the additional advantage of the avoidance of ionizing radiation. The disadvantages of MR are lower spatial resolution, less common availability and higher cost compared to MSCT. Furthermore, recent reports of a condition called nephrogenic systemic fibrosis caused by gadolinium chelates have raised concerns about the safety of gadolinium-based contrast agents (14). 

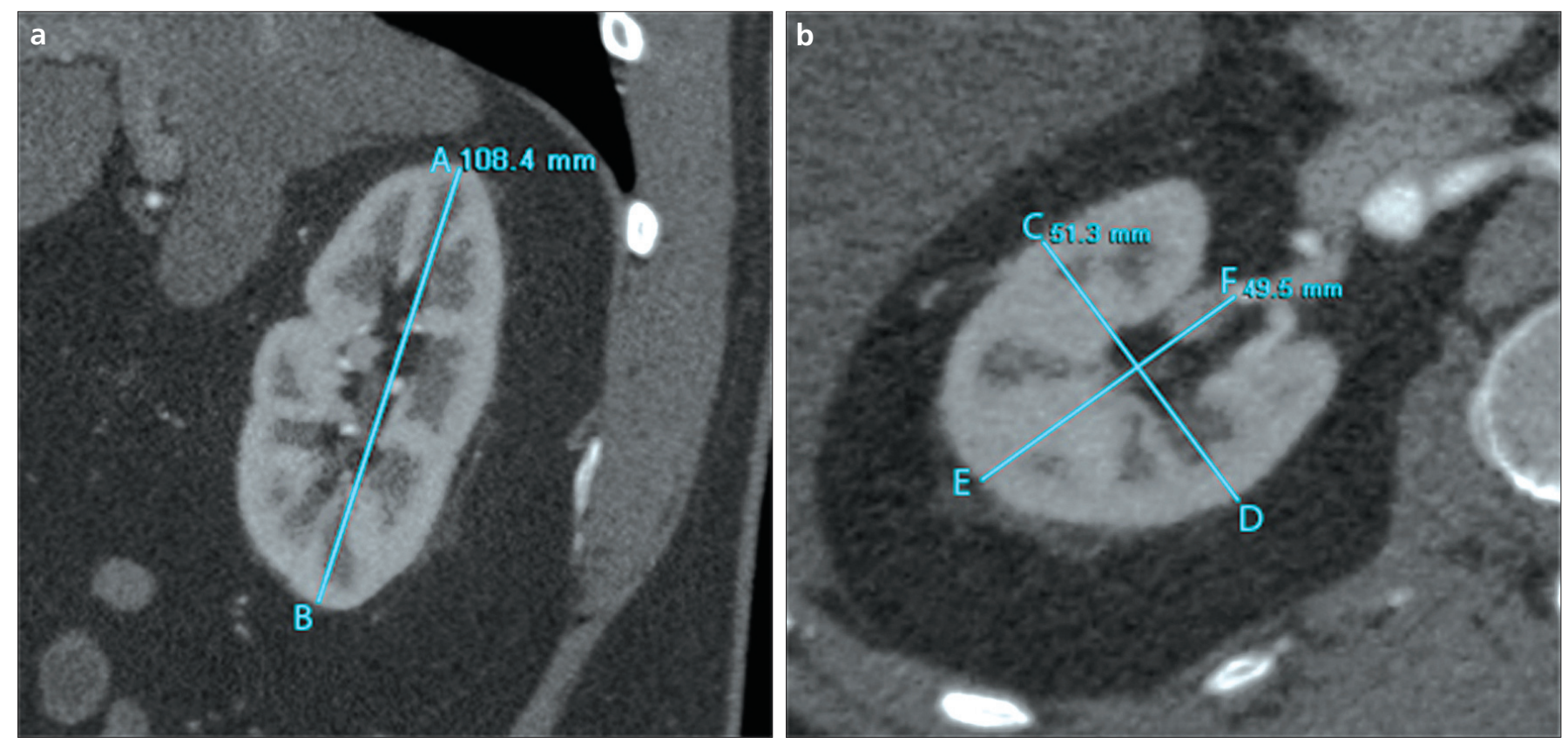

Figure 1. a, b. MDCT of a 53-year-old male. The longest dimensions of kidney length (A-B) on oblique sagittal MPR images (a), the longest dimensions of kidney anteroposterior (C-D) and width (E-F) diameters on oblique axial MPR images (b).

The presence of aRA is diagnosed by direct visualization in each of the modalities mentioned above. Although technological advances have increased the accuracy in determining an aRA, the diagnostic rate still varies depending on the modality used $(9,10,12)$. Indirect parameters that indicate the presence or absence of aRA may facilitate the evaluation of renal vessels. The aim of the present study was to evaluate whether renal dimensions and the diameter of the main renal artery (mRA) could indicate the absence or presence of an aRA in routine radiological practice.

\section{Materials and methods Patients}

The study group consisted of $167 \mathrm{pa}-$ tients (83 women, 84 men; age range, 19-82 years; mean age, $52.4 \pm 13.1$ years) who presented to our radiology department for abdominal CT examinations with various indications between February 2008 and September 2008. The abdominal CT imaging was performed to examine liver lesions $(\mathrm{n}=64)$, pancreatic lesions $(\mathrm{n}=24)$, and abdominal or renal vascular pathology $(\mathrm{n}=79)$. The scanned area was between the level of the diaphragm and the iliac bifurcation in all patients. Patients with a congenital renal abnormality, stenosis in the renal artery, or a history of any renal surgery were not in- cluded in the study group. All of the CT scans were performed during the arterial phase as a standard procedure, and therefore, additional radiation was not an issue.

This study was approved by our institutional review board.

\section{Multislice CT technique}

All CT examinations were performed on a 64-slice CT scanner (Aquillion 64, Toshiba Medical Systems, Otowara, Japan) using the automatic dose modulation technique (Real Exposure Control, Toshiba Medical Systems) in the arterial phase. A total of $90-120 \mathrm{~mL}$ of $300 \mathrm{mg} / \mathrm{mL}$ intravenous contrast material plus a $20-\mathrm{mL}$ chaser bolus was injected through an 18-gauge cannula positioned in an antecubital vein at a flow rate of $4 \mathrm{~mL} / \mathrm{s}$ by a dual head power injector. An automatic triggering system (Sure Start, Toshiba Medical Systems) provided the scanning delay time to obtain an arterial phase image. For this purpose, a single unenhanced low-dose scan was first obtained. Based on this image, a region of interest (ROI) with an area of $1 \mathrm{~mm}^{2}$ was then established by a CT technologist in the lumen of the aorta at the level of the superior mesenteric artery. The patient was instructed to hold his/her breath as soon as the signal density in this region of interest reached a threshold of 180 Hounsfield units, and the scan was then initiated. The scanning delay time was 18-24 seconds after the start of the injection.

\section{Image post-processing and analysis}

A workstation (Vitrea2 version 4.0 workstation, Toshiba Medical Systems) was used to post-process the volumetric MSCT data sets, and the evaluation was performed with multiplanar reformatting (MPR) images using a maximum intensity projection (MIP). Thin $(0.5 \mathrm{~mm})$ and thick $(5 \mathrm{~mm})$ slice thicknesses were used to evaluate the renal arteries.

First, oblique sagittal MPR images parallel to the long axis of the kidney were obtained, and the maximum craniocaudal dimensions were measured (Fig. 1). The oblique axial images were created using these oblique sagittal MPR images, and the maximum transverse and anteroposterior diameters were recorded at the level of the renal hilus in millimeters (Fig. 1). The number of renal arteries supplying each kidney was then evaluated on the MIP images. The number of renal arteries was noted if more than one renal artery was present in a kidney. Oblique coronal, axial and sagittal MIP images were generated for each renal artery to visualize the artery along its route (Fig. 2). At least two of these images provided accurate visualization of the entire length and course of the renal artery 

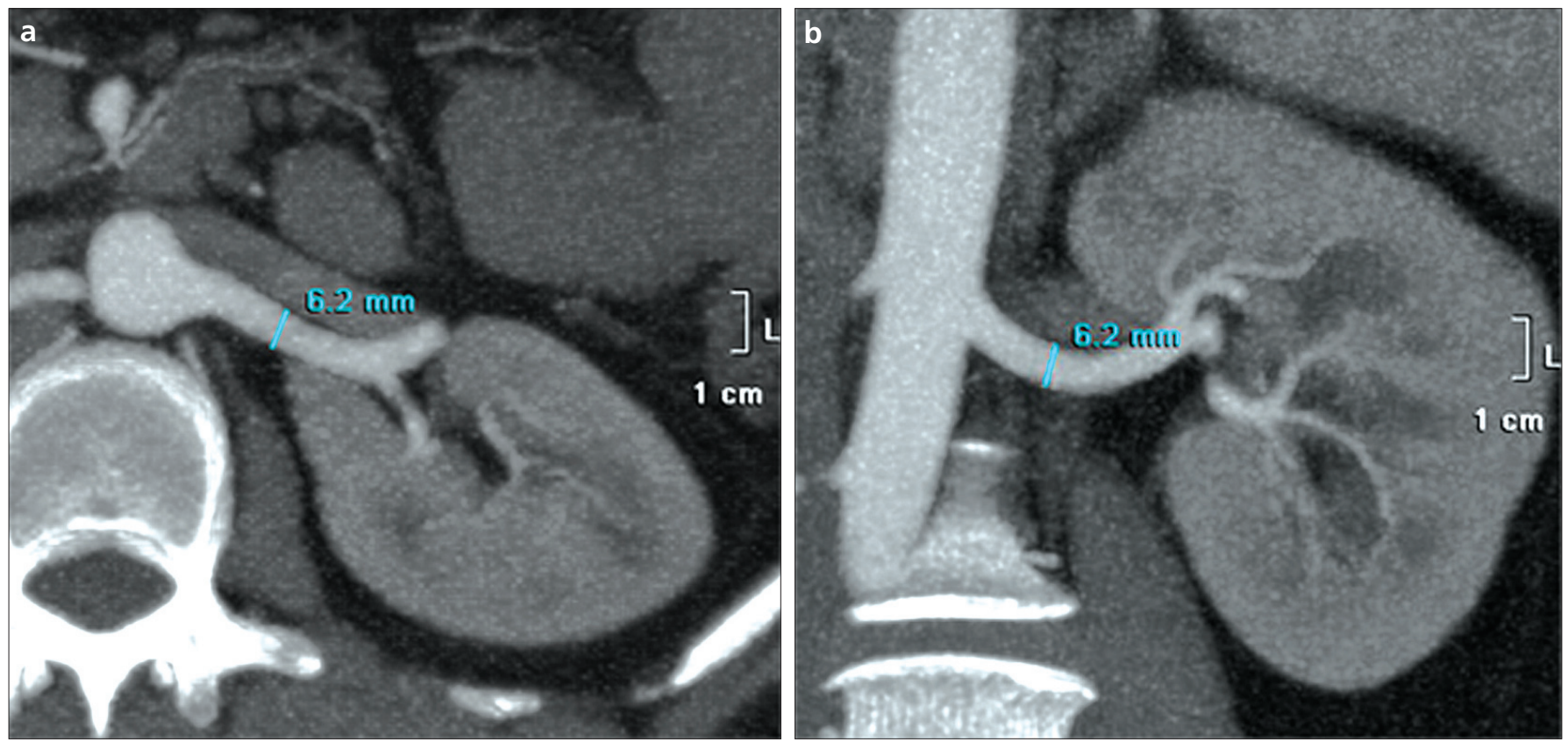

Figure 2. a, b. MDCT of a 39-year-old female. The route of the renal artery on axial (a) and coronal (b) images and renal artery diameter measurement.

and were used to measure the calibers of all arteries (Fig. 2).

The measurement of the RA diameter was performed in the proximal segment of the renal artery at a distance of 1 to $1.5 \mathrm{~cm}$ from the ostium, where the renal artery reaches a uniform width (9). The widest caliber was measured perpendicular to its long axis. If the renal artery demonstrated early branching (which is any branch within $2.0 \mathrm{~cm}$ from the aorta), the caliber was measured before the level of branching. If the kidney has single renal artery, this artery is accepted as an mRA. Whereas, if the kidney has multiple arteries, the largest one is accepted as an mRA.

Finally, we used statistical analyses to create a formula that could be used to determine whether an aRA is absent or present.

\section{Statistical analysis}

The results are expressed as the mean \pm standard deviation (SD), frequency and percent where appropriate throughout the study. The mean differences among the groups with and without aRA were evaluated using the Student's t-test, and the correlations of kidney dimensions with mRA diameter were evaluated by Pearson's correlation analysis.

The prevalence of aRA was compared with the prevalence reported in the lit- erature (25\%) using the exact binomial test for proportions (1-4).

The area under the ROC curve (AUC) analysis was used to determine the best cut-off point for the mRA diameter to predict the aRA. Logistic regression analysis was performed to obtain a function to predict the aRA from the mRA diameter and kidney length. All statistical analyses were performed using the SPSS program (version 15 for Windows), and statistical significance was set at $P<0.05$.

\section{Results}

CT examinations were performed without complications in all patients, and no studies had to be repeated due to technical problems.

\section{Kidney dimensions}

A total of 334 kidneys in 167 patients were examined. The kidney dimensions were as follows: mean length, $108 \pm 11.3 \mathrm{~mm}$; mean width, $49.1 \pm 6.2$ $\mathrm{mm}$; and mean anteroposterior diameter, $47.1 \pm 5.8 \mathrm{~mm}$ (Table 1 ).

Table 1. Mean \pm standard deviation (SD), minimum, maximum and $95 \%$ confidence interval for kidney dimensions (kidney length, width and anteroposterior dimensions), mRA diameter, aRA diameter and statistical correlation of mRA diameter with those parameters

\begin{tabular}{|c|c|c|c|c|}
\hline Measurements & $\begin{array}{c}\text { Mean } \pm \text { SD } \\
(\mathrm{mm})\end{array}$ & $\begin{array}{l}\text { Minimum } \\
(\mathrm{mm})\end{array}$ & $\begin{array}{l}\text { Maximum } \\
(\mathrm{mm})\end{array}$ & $\begin{array}{c}\mathrm{mRA} \\
\text { diameter }\end{array}$ \\
\hline \multicolumn{5}{|l|}{ Kidney dimensions } \\
\hline \multirow[t]{2}{*}{ Length } & $108.03 \pm 11.32$ & 62.5 & 145.0 & $r=0.426$ \\
\hline & & & & $P<0.001$ \\
\hline \multirow[t]{2}{*}{ Width } & $49.06 \pm 6.23$ & 28.8 & 68.6 & $r=0.471$ \\
\hline & & & & $P<0.001$ \\
\hline \multirow[t]{2}{*}{ Anteroposterior } & $47.06 \pm 5.78$ & 30.0 & 66.3 & $r=0.421$ \\
\hline & & & & $P<0.001$ \\
\hline mRA diameter & $5.51 \pm 0.96$ & 1.6 & 8.8 & 1 \\
\hline \multirow[t]{2}{*}{ aRA diameter } & $2.78 \pm 0.98$ & 1.1 & 5.2 & $r=0.080$ \\
\hline & & & & $P=0.492$ \\
\hline
\end{tabular}




\section{The prevalence of $a R A$}

Two hundred fifty-eight (77.2\%) of the 334 kidneys had a single RA (with no aRA), whereas 76 (22.8\%) had one or more aRAs. Of the kidneys with aRAs, 66 kidneys (19.8\%) had one aRA, whereas 10 (3\%) had two aRAs. The prevalence of aRA in our study group $(22.8 \%)$ was similar to that described in the literature $(P=0.376)$.

\section{The MRA and aRA diameters}

The mean diameters for mRA and aRA were $5.51 \pm 0.96 \mathrm{~mm}$ and $2.78 \pm 0.98$ $\mathrm{mm}$, respectively (Table 1 ). The mRA diameters had a positive correlation with kidney dimensions $(\mathrm{r}=0.4-0.5, P$ $<0.001$ ) (Table 1), whereas there was no correlation between the aRA diameter and kidney dimensions.

\section{The features of $M R A$ diameter in kidneys with aRA}

The mRA diameter was smaller in kidneys with an aRA than in those without aRAs $(P<0.001)$ (Table 2 , Fig. $3)$. The highest rates of sensitivity and specificity were obtained when 4.85 $\mathrm{mm}$ was taken as a cut-off point for the mRA diameter to predict the presence an aRA. This cut-off value provided a sensitivity of $54 \%$, a specificity of $87 \%$, a negative predictive value (NPV) of $85 \%$ and a positive predictive value (PPV) of 58\% (AUC, $0.755 ; P<0.001$ ). When $4.15 \mathrm{~mm}$ was taken as a cut-off point for the mRA diameter, the specificity, NPV, and PPV were found to be $99 \%, 80 \%$, and 90\%, respectively, with low sensitivity (26\%). Table 3 shows the sensitivity, specificity, and negative and positive predictive values for three different cut-off points.

\section{The formula to determine the presence of aRAs}

According to the logistic regression results presented in Table 4 , the formula using the kidney length and mRA diameter together had an accuracy of $97 \%$ in showing the absence of aRA and of $38 \%$ in showing the presence of aRA (with an overall accuracy of $85 \%$ ). An increase of $1 \mathrm{~mm}$ in kidney length resulted in an increase in the probability of having an aRA by 1.054 times, and an increase of 1 $\mathrm{mm}$ in $\mathrm{mRA}$ resulted in a decrease in the probability of having an aRA by 0.232 times (Nagelkerke $\mathrm{R}^{2}=0.275$, $P<0.001)$. These logistic regression results can be used to determine the

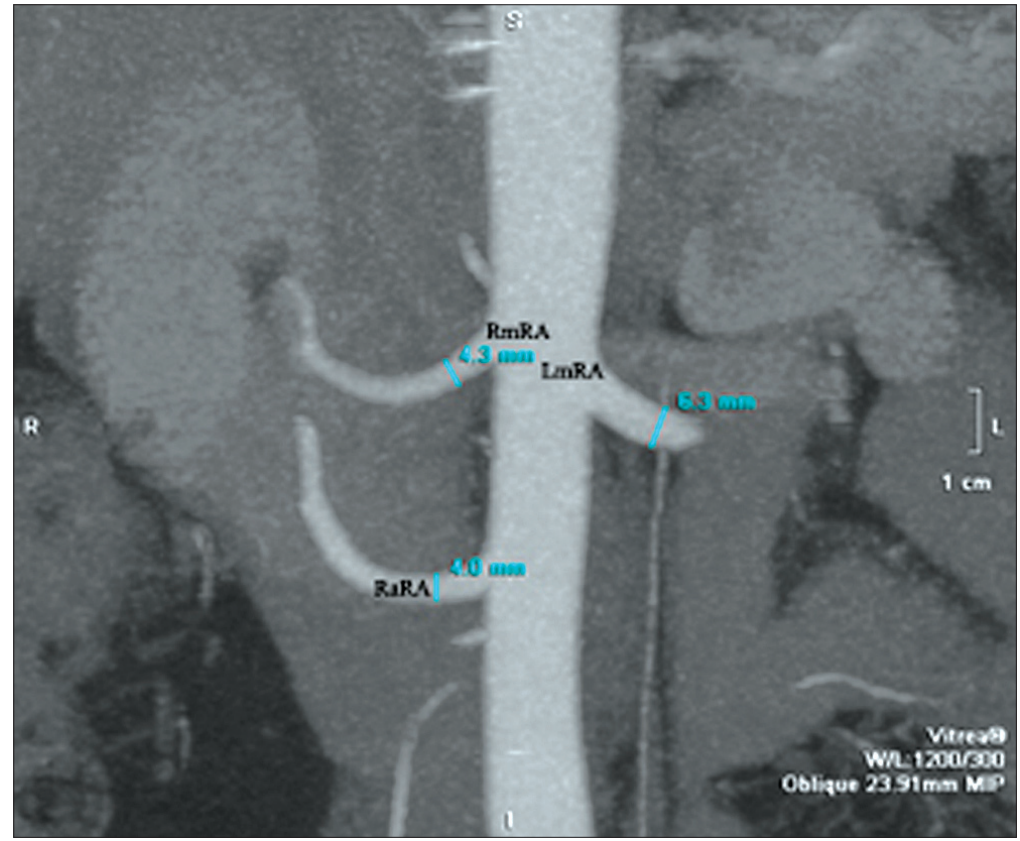

Figure 3. MDCT of a 42-year-old female. Measurements of the diameters of the right main renal artery (RmRA), right accessory artery (RaRA) and left main renal artery ( $L m R A)$. Note that the RmRA is smaller than $4.85 \mathrm{~mm}$, which suggests that it may have an accessory renal artery.

Table 2. The mRA diameter of a kidney without an aRA, with one aRA and with two aRAs

\begin{tabular}{ccccc}
\hline \multirow{2}{*}{$\begin{array}{c}\text { Number of } \\
\text { aRA }\end{array}$} & $\begin{array}{c}\text { Number of } \\
\text { kidneys }\end{array}$ & Mean \pm SD & Minimum & Maximum \\
\cline { 3 - 5 } & 258 & $5.71 \pm 0.83$ & 2.9 & 8.8 \\
1 & 66 & $4.88 \pm 1.07$ & 1.6 & 7.7 \\
2 & 10 & $4.46 \pm 0.97$ & 3.1 & 6.3
\end{tabular}

Table 3. Specificity, sensitivity, and negative and positive predictive values for three different cut-off points for the mRA diameter

\begin{tabular}{ccccc}
\hline $\begin{array}{c}\text { Cut-off point } \\
(\mathrm{mm})\end{array}$ & Sensitivity & Specificity & $\begin{array}{c}\text { Negative } \\
\text { predictive value }\end{array}$ & $\begin{array}{c}\text { Positive } \\
\text { predictive value }^{\mathrm{b}}\end{array}$ \\
\hline 4.85 & $54 \%$ & $87 \%$ & $85 \%$ & $58 \%$ \\
4.65 & $48 \%$ & $91 \%$ & $84 \%$ & $64 \%$ \\
4.15 & $26 \%$ & $99 \%$ & $80 \%$ & $90 \%$ \\
\hline
\end{tabular}

${ }^{a}$ For the negative predictive value calculation, the negative prevalence value is taken as $75 \%$

${ }^{b}$ For the positive predictive value calculation, the positive prevalence value is taken as $25 \%$

Table 4. Results of the logistic regression model using kidney length and mRA diameter

\begin{tabular}{lcccccc}
\hline & & & & \multicolumn{2}{c}{$95 \%$ confidence interval } \\
\cline { 6 - 7 } Parameters & $\beta$ & $\begin{array}{c}\text { Standard } \\
\text { error of } \beta\end{array}$ & $\begin{array}{c}P \\
\text { value }\end{array}$ & $\begin{array}{l}\text { Odds } \\
\text { ratio }\end{array}$ & $\begin{array}{l}\text { Lower } \\
\text { bound }\end{array}$ & $\begin{array}{c}\text { Upper } \\
\text { bound }\end{array}$ \\
\hline mRA diameter & -1.459 & 0.209 & $P<0.001$ & 0.232 & 0.154 & 0.350 \\
Kidney length & 0.052 & 0.015 & $P=0.001$ & 1.054 & 1.023 & 1.086 \\
Nagelkerke $\mathrm{R}^{2}=0.275, P<0.001$ & & & &
\end{tabular}

$\beta$, regression coefficient; Nagelkerke $R^{2}$, Nagelkerke's R square measurement 


$$
\hat{P}=\frac{\exp [0.824-1.459 \times(\text { mRA diameter })+0.052 \times(\text { kidney length })]}{1+\exp [0.824-1.459 \times(\text { mRA diameter })+0.052 \times(\text { kidney length })]}
$$

Figure 4. The probability of having an aRA.

probability of having an aRA according to the formula in Fig. 4.

In this context, the results provide the probability of having an aRA given the mRA diameter and kidney length values. A probability result $(\hat{P})$ equal to or greater than 0.50 indicates the presence of an aRA, whereas a value smaller than 0.50 indicates the absence of an aRA.

\section{Discussion}

The aRA constitutes the most common clinically important renal vascular variant. Previous anatomic, conventional angiographic and CT angiographic reports concerning the renal artery have been typically morphometric studies and have usually investigated the prevalence of an aRA (1, $10,15,16)$. The prevalence of an aRA has been generally accepted to be approximately $25-30 \%$ in different study series $(1-4,7)$.

Knowledge of the presence/absence of an aRA has several advantages in routine daily practice. The most important of these is guidance regarding invasive procedures. Knowledge of the arterial anatomy of the kidney, especially prior to surgeries carried out with a small field of view, such as laparoscopic and retroperitoneoscopic nephrectomy, aids in decisions in planning the nephron-sparing surgery and prevents possible perioperative surprises $(17,18)$. For example, a donor kidney without an aRA is preferred because it allows for an easier surgery for both the donor and the recipient $(6,18,19)$. Bleeding due to an invasive procedure performed on an aRA may result in open laparotomy, whereas a missed case of an aRA may cause an infarct in a transplanted kidney and associated hypertension in the recipient $(12,17)$. It is also necessary to know whether an aRA is present prior to investigating the isolated aRA stenosis in a case with normal mRA hemodynamics (4).

Early generation CT has been accepted as inadequate for the visualization of an aRA (4), because a previous CT angiographic study has reported that the detection of vessels smaller than 2 $\mathrm{mm}$ is limited with this technique (20). In the present study, the smallest renal artery diameter found was $1.1 \mathrm{~mm}$, and this value showed that the stated limitation is overcome by MSCT, especially 64-slice CT, due to the availability of the smallest $(0.5 \mathrm{~mm})$ thickness $(6,7,10)$. In our study with a larger number of patients, the aRA prevalence of $22.8 \%$ was close to the prevalence found in a cadaveric study (2). These findings indicate that MSCT angiography provides results similar to those obtained for the conventional angiography technique $(6,10,18,19)$.

Different radiological methods have shown the aRA directly with high but different degrees of accuracy $(9,10,11$, 20). To increase the diagnostic accura$\mathrm{cy}$, indirect findings may be utilized to determine the presence/absence of an aRA. In a study performed to evaluate indirect findings to predict the presence of an aRA, Aytac et al. (9) have found that the mRA diameter is significantly smaller in the presence of an aRA. Because this study group included only patients with normal kidney length dimensions (80-120 mm), the generalization of these findings to other kidneys remains unclear. However, the same finding was verified in our study with no limitations of the kidney dimensions and a larger number of patients with and without aRA (Table 2).

The finding of a smaller mRA diameter in kidneys with an aRA, in parallel to the study reported by Aytac et al. (9), has led to the question of whether a mRA diameter cut-off value can be determined for such kidneys. The highest rates of sensitivity and specificity for determining the aRA (54\% sensitivity, $87 \%$ specificity, $85 \% \mathrm{NPV}$, and $58 \%$ PPV) were obtained when 4.85 $\mathrm{mm}$ was taken as a cut-off point for the mRA diameter (Table 3). However, despite the high specificity and NPV values, the sensitivity and PPV values for this cut-off value were not high enough to provide an idea about the presence of an aRA of a kidney during routine investigation.
The $4.65 \mathrm{~mm}$ cut-off value for the mRA diameter reported in the study by Aytac et al. (9) showed 80\% sensitivity and $80.5 \%$ specificity. Using this mRA diameter cut-off value $(4.65 \mathrm{~mm})$ in the present study provided high specificity (91\%) but low sensitivity values (Table 3). However, when a cut-off value of $4.15 \mathrm{~mm}$ was used for both Aytac et al.'s (9) and the present data, the sensitivity decreased significantly but the specificity increased to 99\%. In the present study, the PPV and NPV values for a cut-off value of $4.15 \mathrm{~mm}$ were $90 \%$ and $80 \%$, respectively. This result indicates that it is possible to state that "aRA is present" with 90\% accuracy when the mRA diameter is $4.15 \mathrm{~mm}$ or smaller and that "aRA is absent" with 80\% accuracy when the mRA diameter is larger than $4.15 \mathrm{~mm}$ in a particular kidney. The PPV and NPV values calculated from data reported by Aytac et al. were similarly high (96\% and $86 \%$, respectively). Although the best cut-off value, patient groups and radiological techniques used (DSA, MSCT) differed between the two studies, a common finding was the high probability of the presence of an aRA when the mRA diameter was equal to or smaller than $4.15 \mathrm{~mm}$. This result suggests that the discovery of a mRA diameter of 4.15 $\mathrm{mm}$ or less during radiological investigations indicates a high probability of the presence an aRA and should lead to more detailed studies regarding the presence of an aRA in that kidney. This information is important; however, only $23(6.9 \%)$ of the 334 kidneys assessed in our study demonstrated such mRA values, and thus, such findings are not a common occurrence in routine practice.

Our inability to detect a mRA diameter cut-off value with sufficiently high specificity and sensitivity together to detect an aRA has limited the utility of mRA diameter values alone in routine practice. Logistic regression analysis to detect the presence/absence of aRA has shown that the mRA diameter and longitudinal kidney length are the influential factors. Therefore, we developed a formula comprising these two variables, which can be easily and quickly measured in routine practice. We found a low degree of accuracy when this formula was used to detect the presence of an aRA but a very high degree of accuracy (97\%) when it was used to detect the absence of an aRA. 
The longitudinal kidney length and mRA diameter, which can be quickly measured during renal artery evaluations in routine practice, revealed the absence of an aRA with 97\% accuracy when the result was less than 0.50 .

The information obtained regarding the presence/absence of an aRA using the indirect findings described above does not eliminate the requirement for conventional CT or MR angiography to evaluate the renal arteries before invasive procedures such as nephron-sparing renal tumor surgery or renal transplantation. However, these indirect findings may decrease the number of additional investigations needed to exclude isolated aRA stenosis (although rare), as they indicate the presence or absence of aRA. These indirect findings may also supply preliminary information to the radiologist, thus decreasing the study evaluation time and facilitating confirmation of the decision.

The main limitation of our data is that they were not verified by other radiological methods. We think that the measurements obtained herein can be considered as actual dimensions because we used MSCT together with isotropic data acquisition, which provides images that closely mimic the actual state. In the literature, the accuracy of MSCT angiography has been described to be similar to that of DSA, and the prevalence of aRA in the present study was similar to that described in the literature. However, these MSCT values cannot be used to replace other radiological methods in an equivalent manner, such as US, MRI or DSA, due to differences between the techniques. The demonstration of a correlation with other investigative techniques could increase the reliability of the study results; however, this would require that the patients undergo a greater number of investigations and would probably decrease the number of study subjects.

Another limitation was that although the developed formula was almost $100 \%$ accurate in predicting the absence of an aRA, this database was limited to our own patient group, albeit with a large number of patients. This formula needs to be used in differ- ent patient groups and with different radiological modalities in other studies to test the validity of the formula.

In conclusion, the measurement of the mRA diameter can provides clues regarding the presence of an aRA, and an aRA can be said to be present with an accuracy of $90 \%$ in a kidney with a mRA diameter equal to or smaller than $4.15 \mathrm{~mm}$. Using the formula that employs the mRA diameter and kidney length, as defined in our study for the first time, enables us to determine the absence of an aRA with almost 100\% accuracy. The use of these results in aRA evaluation can decrease the assessment duration and facilitate radiologist decisions concerning the requirement for advanced investigations, depending on the indication, by providing quick and reliable preliminary information regarding the presence or absence of an aRA.

\section{References}

1. Urban BA, Ratner LE, Fishman EK. Threedimensional volume-rendered CT angiography of the renal arteries and veins: normal anatomy, variants, and clinical applications. Radiographics 2001; 21:373-386.

2. Williams PL, Warwick R, Dyson $M$, Bannister LH. Gray's anatomy. 37th ed. London: Churchill Livingstone, 1989; 1407-1409.

3. Neri E, Caramella D, Bisogni C, et al. Detection of accessory renal arteries with virtual vascular endoscopy of the aorta. Cardiovasc Intervent Radiol 1999; 22:1-6.

4. Saba L, Sanfilippo R, Montisci R, Conti $M$, Mallarini G. Accessory renal artery stenosis and hypertension: are these correlated? Evaluation using multidetector-row computed tomographicangiography. Acta Radiol 2008; 3:278-284.

5. Coll DM, Uzzo RG, Hertz BR, et al. 3-dimensional volume rendered computed tomography for preoperative evaluation and intraoperative treatment of patients undergoing nephron sparing surgery. J Urol 1999; 161:1097-1102.

6. Raman SS, Pojchamarnwiputh S, Muangsomboon K, Schulam PG, Gritsch HA, Lu DSK. Utility of 16-MDCT angiography for comprehensive preoperative vascular evaluation of laparoscopic renal donors. AJR Am J Roentgenol 2006; 186:1630-1638.

7. Kawamoto S, Montgomery RA, Lawler LP, Horton KM, Fishman EK. Multidetector CT angiography for preoperative evaluation of living laparoscopic kidney donors. AJR Am J Roentgenol 2003; 180:1633-1638.
8. Rastogi N, Sahani D, Blake MA, Ko DC, Mueller PR. Evaluation of living renal donors: accuracy of three-dimensional 16 section-CT. Radiology 2006; 240:136-144.

9. Aytac SK, Yigit H, Sancak T, Ozcan H. Correlation between the diameter of the main renal artery and the presence of an accessory renal artery. J Ultrasound Med 2003; 22:433-439.

10. Türkvatan A, Özdemir M, Cumhur T, Ölçer T. Multidetector CT angiography of renal vasculature: normal anatomy and variants. Eur Radiol 2009; 19:236-244.

11. Kock MCJ, Lijzermans JNM, Visser K, et al. Contrast-enhanced MR angiography and digital subtraction angiography in living renal donors: diagnostic agreement, impact on decision making, and costs. AJR Am J Roentgenol 2005; 185:448-456.

12. Gluecker TM, Mayr M, Schwarz J, et al. Comparison of CT angiography with MR angiography in the preoperative assessment of living kidney donors. Transplantation 2008; 86:1249-1256.

13. Jha RC, Korangy SJ, Ascher SM, Takahama J, Kuo PC, Johnson LB. MR angiography and preoperative evaluation for laparoscopic donor nephrectomy. AJR Am J Roentgenol 2002; 178:1489-1495.

14. Prince MR, Zhang $H$, Morris $M$, et al. Incidence of nephrogenic systemic fibrosis at two large medical centers. Radiology 2008; 248;807-816.

15. Satyapal KS, Haffejee AA, Singh B, Ramsaroop L, Robbs JV, Kalideen JM. Additional renal arteries: incidence and morphometry. Surg Radiol Anat 2001; 23:33-38.

16. Beregi JP, Mauroy B, Willoteaux S, Mounier-Vehier C, Remy-Jardin M, Francke JP. Anatomic variation in the origin of the main renal arteries: spiral CTA evaluation. Eur Radiol 1999; 9:1330-1334.

17. Makiyama $\mathrm{K}$, Tanabe $\mathrm{K}$, Ishida $\mathrm{H}$, et al. Successful renovascular reconstruction for renal allografts with multiple renal arteries. Transplantation 2003; 75:828-832.

18. Kawamoto S, Montgomery RA, Lawler LP, Horton KM, Fishman EK. Multi-detector row CT evaluation of living laparoscopic renal donors prior to laparoscopic nephrectomy. Radiographics 2004; 24: 453-466.

19. Mandal AK, Cohen C, Montgomery RA, Kavoussi LR, Ratner LE. Should the indications for laparoscopic live donor nephrectomy of the right kidney be the same as for the open procedure? Anomalous left renal vasculature is not a contraindication to laparoscopic left donor nephrectomy. Transplantation 2001; 71:660-664.

20. Platt JF, Ellis JH, Korobkin M, Reige K. Helical CT evaluation of potential kidney donors: findings in 154 subjects. AJR Am J Roentgenol 1997; 169:1325-1330. 